\title{
A Comparative Study of Pretrained Language Models on Thai Social Text Categorization
}

\author{
Thanapapas Horsuwan ${ }^{1}$, Kasidis Kanwatchara ${ }^{1}$, \\ Peerapon Vateekul ${ }^{1}$, and Boonserm Kijsirikul ${ }^{1}$ \\ Department of Computer Engineering, Faculty of Engineering, \\ Chulalongkorn University, Bangkok, Thailand \\ \{thanapapas.h, kanwatchara.k\}@gmail.com \\ \{peerapon.v, boonserm.k\}@chula.ac.th
}

\begin{abstract}
The ever-growing volume of data of user-generated content on social media provides a nearly unlimited corpus of unlabeled data even in languages where resources are scarce. In this paper, we demonstrate that state-of-the-art results on two Thai social text categorization tasks can be realized by pretraining a language model on a large noisy Thai social media corpus of over 1.26 billion tokens and later fine-tuned on the downstream classification tasks. Due to the linguistically noisy and domain-specific nature of the content, our unique data preprocessing steps designed for Thai social media were utilized to ease the training comprehension of the model. We compared four modern language models: ULMFiT, ELMo with biLSTM, OpenAI GPT, and BERT. We systematically compared the models across different dimensions including speed of pretraining and fine-tuning, perplexity, downstream classification benchmarks, and performance in limited pretraining data.
\end{abstract}

Keywords: language model $\cdot$ pretraining $\cdot$ Thai social media $\cdot$ comparative study $\cdot$ data preprocessing

\section{Introduction}

Social networks are active platforms rich with a quickly accessible climate of opinion and community sentiment regarding various trending topics. The growth of the online lifestyle is observed by the bustling active communication on social media platforms. Opinion-oriented information gathering systems aim to extract insights on different topics, which have numerous applications from businesses to social sciences. Nevertheless, existing NLP researches on utilizing these abounding noisy user-generated content have been limited despite its potential value.

First introduced in [7, pretrained language models (LMs) have been a topic of interest in the NLP community. This interest has been coupled with works reporting state-of-the-art results on a diverse set of tasks in NLP. In light of the notable benefits of transfer learning, we chose to compare four renowned LMs: ULMFiT [9], ELMo with biLSTM [14, OpenAI GPT [16, and BERT [8]. To the best of our knowledge, our work is the first comparative study conducted 
on pretrained LMs in Thai language. Our LMs were trained in a three-stage process as per suggested in 9]: LM pretraining, LM fine-tuning, and classifier fine-tuning. The goal of unsupervised pretraining is to find a good initialization point to capture the various general meaningful aspects of a language. Befitting Thai language with resource scarcity, we expect that pretraining user-generated content would serve as a solid basis for transfer learning to downstream tasks.

Pantip is the largest Thai internet forum with a huge active community where a diverse range of topics are discussed. The variability of surplus examples from Pantip covers the basic linguistic syntax of Thai language while maintaining the colloquial and noisy nature of online user-generated content. In this paper, we investigate and compare the capability of each LM to capture the relevant features of a domain-specific language via pretraining copious unlabeled data from user-generated content.

The main contributions of this paper are the following:

- We developed unique data preprocessing techniques for Thai social media.

- We pretrained ULMFiT, ELMo, GPT, and BERT on a noisy Thai social media corpus much larger than the existing Thai Wikipedia Dump.

- We compared the language models across different dimensions including speed of pretraining and fine-tuning, perplexity, downstream classification benchmarks, and performance in limited pretraining data.

- Our pretrained models and code can be obtained upon request to the corresponding authors

This paper is organized as follows. Our data preprocessing techniques are explained in Section 2 and the LMs used for pretraining are briefly described in Section 3. The datasets used in this paper are described in Section 4 and Section 5 explains our hyperparameters and evaluation metrics. The results are reported in Section 6 and finally concluded in Section 7.

\section{Our Data Preprocessing for Thai Social Media}

Data preprocessing is one of the most important phases in improving the learning comprehension of the LMs. If much irrelevant and redundant information introduces unwanted noise in the training corpus, it is difficult for the models to discover knowledge during the training phase. This is especially true for unfiltered data from user-generated content on social media, where it requires specific methods of data preprocessing unique to the domain.

The Thai webboard Pantip allows members to freely create threads as long as it conforms to a list of actively regulated etiquette. The colloquial nature of the data posted allows for huge amounts of noise to be introduced in the data, such as ASCII arts, language corruption (ภาษาวิบัติ), irregular spacing, misspelling, character repetition, and spams [10. The unpredictable noise in the data substantially increases the vagueness of word boundary, which already is a problem in formal Thai language [4. Additionally, Thai word segmentation is dependent on context. A famous example is the compound word 'ตากลม', which 
can be either split into '[ตา][กลม]' or '[ตาก][ลม]'. Both are grammatically correct when used within their corresponding context. To ease the impact of the issues, the data preprocessing approaches we employed are as follows:

1. Length Filtering To select meaningful threads to the LM, threads with a title and with a body of more than 100 characters were selected.

2. Language Filtering An n-gram-based text categorization library langdetect 1 was used to filter out the threads that are not labeled as Thai language.

3. General Preprocessing before Tokenization Inspired by [93], the techniques include fixing HTML tags, removing duplicate spaces and newlines, removing empty brackets, and adding spaces around ' ' and '\#'. In addition, character order in Thai language may be typed in a different sequence but visually rendered in the same way. This is due to the fact that vowels, diphthongs, tonal marks, and diacritics may sometimes be physically located above or below the base glyph-allowing different sequential orders to appear visually equivalent. Thus, normalizing the character order is required for the machine to understand the seemingly similar tokens.

4. Customized Preprocessing before Tokenization We also developed and customized techniques suitable for Thai social media. Last character repetition is a common behavior of Thai people analogous to prolonging the vowel sounds of a word in spoken language to emphasize certain emotions. We truncate the word and follow it by a special token. pyThaiNLP [15] adopted a similar technique but we implemented minor modifications of space addition following the token for better tokenization results. Likewise, a special token is used for word repetitions similar to [9] preprocessing technique, which at the time this technique has not been widely used in Thai language preprocessing. Since Thai is a language without word boundaries, our algorithm recognizes words as any character sequence of more than 2 characters with more than 2 repetitions of that sequence. All types of repetitions are truncated to 5 as it provides no higher emotional impact and to limit the vocabulary size.

In addition, we also propose 2 new preprocessing methods: a special token for any numeric strings and a special token for laughing expressions.

We replaced all strings related to numbers with a special token: general numbers, masked and unmasked phone numbers, Thai numbers, date and time, masked prices, and numbers of special forms. Although differentiating the numbers provide some semantic value, the sparsity of the information would most likely make these numbers tail out of vocabulary (OOV) tokens. We believe that this preprocessing method would allow the language models to more generally understand how numbers are used in text.

In an online environment, Thai people often express laughter in written language with an onomatopoeia, utilizing the repetition of ' 5 ' followed by an optional ' + '. This is due to the fact that the Thai pronunciation of ' 5 ' is 'ha'. We replaced all tokens with more than 3 consecutive ' 5 ' and an optional ' + ' with a special laugh token. Although this may have a minor effect on actual

\footnotetext{
${ }^{1}$ github.com/fedelopez77/langdetect
} 
numbers, this onomatopoeia is very commonly used in Thai online context and it is important for the model to learn this special token. An example is provided in Table 1 for clarification.

Table 1. An example of our preprocessing method. [CREP] and [LAUGH] are special tokens used for character repetition and laughing respectively.

\begin{tabular}{c|cc}
\hline & Before & After \\
\hline Thai & ฉันชอบมันมากกกก555555+ ฉันชอบมันมาก [CREP] 4 [LAUGH] \\
Translated & I like it a lotttt hahahahaha ${ }^{1}$ I like it a lot [CREP] 4 [LAUGH] \\
\hline
\end{tabular}

1 ' 5 ' is pronounced 'ha' in Thai

5. Tokenization We used the pyThaiNLP 15] default tokenizer, which is a dictionary-based Maximum Matching with Thai Character Cluster. However, we created our own aggregated dictionary for tokenization to improve the tokenization accuracy for colloquial user-generated content. The dictionary 2 is compiled from various sources of data, including general words, abbreviations, transliterations, named entities, and self-annotated Thai slangs and commonly used corrupted language. This includes word variants like ฮะ ฮาฟ ฮับ ฮัฟ คร้าบ ค้าฟ ค้าบ คับ ครัช which are all word variants of the suffix to indicate formality ครับ. The vocabulary is built from the most common $80 \mathrm{k}$ tokens.

6. General Preprocessing after Tokenization: Following [9] and [3], some general preprocessing techniques after tokenization were used. This includes ungrouping the emoji's from text, and to lowercase all English words.

7. Spelling Correction In an effort to reduce the number of unnecessary tokens sprouting from incorrectly spelled words, we compiled a list of commonly misspelled word mappings aggregated from various sources. We corrected and standardized the vocabulary used. This is an important task due to the free and lax nature of the corpus, where a single word may be represented in different variants or misspelled and abbreviated into various tokens. Note that not all replacements can be made due to the collision of actual vocabularies and the limited comprehensiveness of the list.

\section{Pretrained Language Models in Our Study}

\subsection{Universal Language Model Fine-tuning (ULMFiT)}

A single model architecture that is used for both LM pretraining and downstream fine-tuning was first introduced in ULMFiT [9. This allows the weights learnt during pretraining to be reused instead of constructing a new task-specific model. Howard and Ruder suggested that LM overfits to small datasets and suffers catastrophic forgetting when directly fine-tuned to a classifier. Hence, the

\footnotetext{
${ }^{2}$ The dictionary is referenced at our GitHub https://github.com/Knight-H/thai-lm
} 
ULMFiT approach was proposed to attempt to effectively fine-tune the AWDLSTM [1] model. ULMFiT is a 3-stage training method consisting of LM pretraining, LM fine-tuning, and classifier fine-tuning. They also proposed novel techniques such as discriminative fine-tuning, gradual unfreezing, and slanted triangular learning rates for stable fine-tuning.

\subsection{Embeddings from Language Models (ELMo)}

Traditional monolithic word embeddings such as word2vec [12] and GloVe [13] fails to model context-dependent meanings of a word. Hence, ELMo [14 produces contextualized word embeddings by utilizing a pretrained biLM as a fixed feature extractor and incorporate its embedding representation as features into another task-specific model for downstream tasks. The authors suggested that combining the internal states of the LSTM layers allows for rich contextualized word representations on top of the original context-independent word embeddings.

\subsection{Generative Pretrained Transformer (GPT)}

Sequential computation models used in sequence transduction problems [5]6[17] forbid parallelization in the training examples. The transformer [18] is the first transduction model based solely on self-attention to draw global dependencies between input and output, eliminating the use of recurrence and convolutions. OpenAI introduced GPT [16] by extending the idea to multi-layer transformer decoder for language modeling. Additionally, LM fine-tuning and classifier finetuning are done simultaneously by using LM as an auxiliary objective. The authors suggested that this improves the generalization of the supervised model and accelerates convergence.

\subsection{Bidirectional Encoder Representations from Transformers (BERT)}

ULMFiT [9] and GPT [16] use a unidirectional forward architecture while ELMo [14 uses a shallow concatenation of independently trained forward and backward LMs. With criticism on the standard unidirectional LMs as suboptimal by severely restricting the power of pretrained representations, BERT [8] was proposed as a multi-layer transformer encoder designed to pretrain deep bidirectional representations by jointly conditioning on both left and right context in all layers. Since the standard autoregressive LM pretraining method is not suitable for bidirectional contexts, BERT is trained on masked language modeling (MLM) and next sentence prediction (NSP) tasks. MLM masks $15 \%$ of the input sequences at random and the task is to predict those masked tokens, requiring more pretraining steps for the model to converge. The output of the special first token is used to compute a standard softmax for classification tasks. 


\section{Dataset}

\subsection{Pretraining Dataset}

To collect our Thai social media corpus data, we extracted non-sensitive information from all threads from Pantip.com since $1^{\text {st }}$ January 2013 up until $9^{\text {th }}$ February 2019 using our implementation of the Scrapy Framework [2]. A total of $8,150,965$ threads were extracted. As discussed in Section 2, data preprocessing techniques are applied to the corpus. Length filtering and language filtering filtered down the threads to 5,524, 831 and 5, 487, 568 respectively. After preprocessing, tokenization, and postprocessing the data, we divided our pretraining dataset into 3 parts: 5, 087, 568 threads for training, 200, 000 threads for validation, and 200,000 threads for testing. The train dataset, validation dataset, and test dataset has a total of 1,262,302,083 tokens, 4,701,322 tokens, and $4,588,245$ tokens respectively. By comparison, our pretrain dataset is more than 31 times larger than the Thai Wikipedia Dump with respect number of tokens, which is only on the order of $40 \mathrm{M}$ tokens for the training set.

\subsection{Benchmarking Dataset}

Two Thai social text classification tasks were chosen to benchmark the models for extrinsic model evaluation as shown in Table 2. Since both are originally Kaggle competitions, the Kaggle evaluation server will be used for benchmarking.

Wongnai Challenge: Rating Review Prediction First initiated as a Kaggle competition, the Wongnai Challenge is to create a multi-class classification sentiment prediction model from textual reviews. As an emerging online platform in Thailand, Wongnai holds a large user base of over 2 million registered users with a surplus of user-written reviews accompanied by a rating score ranging from 1 to 5 stars. This is challenging due to the varying user standards, corresponding to shifting weighted importance of each sentiment in mixed reviews.

Wisesight Sentiment Analysis The Wisesight Sentiment Analysis is a private Kaggle competition where the task is to perform a multi-class classification on 4 categories: positive, negative, neutral, and question. Wisesight, a social data analytics service provider, provides data from various social media sources with various topics on current internet trends. It should be noted that the topics and the source of the data are much more diverse than that of Wongnai.

\section{Experimental Setup}

\subsection{Implementation Details}

ULMFiT We used the same model hyperparameters as the popular Thai GitHub repository thai2fit [3]: the base model is a 4-layer AWD-LSTM with 1,550 hidden activation units per layer and an embedding size of 400. A BPTT batch size of 70 was used. We applied dropout of 0.25 to output layers, 0.1 to RNN layers, 0.2 to input embedding layers, 0.02 to embedding layers, and weight dropout of 0.15 to the RNN hidden-to-hidden matrix. 
Table 2. Datasets, tasks, number of classes, train and test examples, and the average example length measured in tokens. The OOV rate is measured with respect to the original vocabulary of the pretraining corpus.

\begin{tabular}{llccccc}
\hline Dataset & Task & Classes Train Test & OOV & Average Length \\
\hline Wongnai & Sentiment Classification & 5 & $40 \mathrm{k}$ & $6.2 \mathrm{k}$ & $0.71 \%$ & $126 \pm 124$ \\
Wisesight & Sentiment Classification & 4 & $26.7 \mathrm{k}$ & $3.9 \mathrm{k}$ & $2.69 \%$ & $27 \pm 44$ \\
\hline
\end{tabular}

ELMo We used the same biLM architecture from the original implementation [14] with all default hyperparameters, where the LM is a 2-layer biLSTM with 4096 units and 512 dimension projections with another static character-based representations layer with convolutional filters. For both downstream tasks, a 3-layer biLSTM was used with 256 hidden units as the task-specific model.

GPT Default configurations of [16 were used. The resulting model has 12 layers of transformer each with 12 self-attention heads and 768-dimensional states. We used learnt position embeddings and a maximum sequence length of 256 tokens.

BERT We used the publicly available $B E R T_{B A S E}$ unnormalized multilingual cased model, which has a hidden size of 768,12 self-attention heads, and 12 transformer blocks. Note that the $B E R T_{B A S E}$ was chosen to have identical hyperparameters as GPT for comparative purposes.

\subsection{Evaluation Metrics}

A total of 4 tasks were evaluated: the proposed data preprocessing technique in Section 2, LM pretraining, LM fine-tuning, and classifier fine-tuning. We chose to benchmark on the easiness to train each model (speed and number of epochs), the intrinsic evaluations (perplexity), and the extrinsic evaluations (downstream classification tasks). In addition, an ablation study of limited corpus data is compared to see the performance of each model in smaller data scenarios.

Data Preprocessing To benchmark the quality of our unique data preprocessing techniques for Thai social media corpus, we sampled a thread from each dataset and request expert Thai native speakers to help tokenize the samples. At the time of writing, there is no standard corpus for benchmarking the task of colloquial Thai word segmentation. Each character in the thread is labeled as 1 (beginning of word) or 0 (intra-word character). The precision, recall, and F1 score is calculated based on the performance of segmenting each character, where true positives are the correctly segmented beginning of word. The default pyThaiNLP tokenizer [15] Maximum Matching (newmm) is compared between with and without our data preprocessing methods. Unfortunately, labeling tokenization dataset in Thai language requires large amount of effort. Therefore, more extensive experiments will be conducted in the future. 
Language Model Pretraining Pretraining a language model is the most expensive process in the transfer learning workflow. This task is generally performed only once before fine-tuning on a target task. With minimal hyperparameter tuning, we evaluated the pretraining process on: (1) the speed of training in each epoch and (2) the intrinsic perplexity value. Although with the ambiguity that comes with intrinsic metrics, perplexity is one of the traditional methods in LM evaluation. It measures the confidence of the model on the observed sequence via exponentiation of the cross-entropy loss, where cross-entropy loss is defined as the negative sum of the mean LM log-likelihood. Note that this definition applies to different levels of granularity. Due to resource constraints, each model was pretrained for a fixed number of epochs. An NVIDIA P6000 is used to pretrain each model, and the appropriate batch size was selected such that it maximizes the GPU VRAM of 24 GB. The models were trained for 3 epochs and the best performing model was selected. However, since BERT trains using MLM and is able to learn just $15 \%$ of the corpus during 1 epoch, we decided to train for the standard 1 million steps $[8$ (equivalent to around 6.5 epochs).

Language Model Fine-tuning Each model was benchmarked on the number of epochs used and the total time until convergence. This process aims to learn the slight differences in data distribution of the target corpus. The models overfit easily due to the modest size of the corpus, thus each LM was fine-tuned until early stopping.

Classifier Fine-tuning In this paper, we reported each downstream task performance following the metric used in each Kaggle competition. Wongnai Rating Review Challenge and Wisesight Sentiment Analysis both use classification accuracy for evaluation, which is calculated by the proportion of correctly classified samples out of all the samples. Kaggle ranks the competitors' final standings with the private score, hence this will be used as the benchmark.

\section{Results}

In this section, we first report the results of our unique preprocessing methods, followed by the results of pretraining the data. We then compare the results of ULMFiT, ELMo, GPT, and BERT with the previous state-of-the-art models in the Thai NLP research community from the Kaggle competition benchmarks.

\subsection{Data Preprocessing}

Results are shown in Table 3, where our preprocessing method allows the default pyThaiNLP maximum matching (MM) tokenizer to more precisely segment noisy social media data. This is due to the lower false positive tokens segmented by the noisiness of the data, where most of the spams and repetitions are preprocessed correctly. With more comprehensive vocabulary, it allows the tokenizer to segment short colloquial words more accurately. Note that this does not account 
for the supposed increased comprehension of the models from standardizing the data.

Table 3. Tokenization Precision, Recall, and F1-score

\begin{tabular}{lccl}
\hline Tokenizer & Precision & Recall & F1-score \\
\hline MM+Our Preprocessing & $\mathbf{9 5 . 8 3 \%}$ & $98.65 \%$ & $\mathbf{9 7 . 2 2 \%}$ \\
MM & $96.04 \%$ & $\mathbf{9 7 . 3 9 \%}$ & $96.71 \%$ \\
\hline
\end{tabular}

\subsection{Language Model Pretraining}

From Table 4. AWD-LSTM with ULMFiT requires the least amount of time per epoch and the least total time, 100 hours and 33 hours respectively. Due to resource scheduling limitations, ELMo is trained with 2 P6000 GPUs, making the total time and the time per epoch much lower than the supposed value. With character-level convolutions and character-based operations, ELMo training time should be the longest amongst all the LMs. Transformer-based models require time around more than 1.5x of ULMFiT.

Table 4. Model Pretraining Time. $t_{\text {epoch }}$ is the time used per epoch.

\begin{tabular}{l|l}
\hline Model & $t_{\text {epoch }}$ \\
\hline ULMFiT & $\mathbf{3 3} \mathbf{~ h r}$ \\
biLM(ELMo) (2 GPU) & $52 \mathrm{hr}$ \\
GPT seqmax $=256$ & $55 \mathrm{hr}$ \\
BERT seq max $_{\text {max }}=256$ & $49 \mathrm{hr}$ \\
\hline
\end{tabular}

The training loss and perplexity are shown in Table 5. BERT has the lowest word-level cross-entropy loss with 15.3857 MLM perplexity. This is expected due to the difference of the MLM prediction task with fully visible beginning and ending context, providing more contextual information to predict the masked word as compared with traditional forward and backward models. In the domain of traditional autoregressive models, GPT has a lower perplexity than ULMFiT. ELMo is not compared to other models due to prediction granularity difference and is reported as is.

\subsection{Language Model Fine-tuning}

All the language models are fine-tuned with the target corpus until they give the best result with respect to the validation loss. An NVIDIA P6000 is used for each model and the time required is presented in Table 6. Transformer-based models are shown to overfit quicker than LSTM-based models. 
Table 5. Training Loss and Perplexity After Pretraining

\begin{tabular}{lcr}
\hline Model & Loss & Perplexity \\
\hline ULMFiT & 3.5281 & 34.0603 \\
GPT $s e q_{\max }=256$ & 3.1735 & 23.8913 \\
BERT MLM $s e q_{\max }=256$ & $\mathbf{2 . 7 3 3 4}$ & $\mathbf{1 5 . 3 8 5 7}$ \\
\hline biLM(ELMo) (Character-Level) & 1.7140 & 5.5512 \\
\hline
\end{tabular}

Table 6. Language Model Fine-tuning Time. $t_{\text {total }}$ is the total time used and $t_{\text {epoch }}$ is the time used per epoch.

\begin{tabular}{|c|c|c|c|c|c|c|}
\hline \multirow[t]{2}{*}{ Model } & \multicolumn{3}{|c|}{ Wisesight } & \multicolumn{3}{|c|}{ Wongnai } \\
\hline & \#Epoch & $t_{\text {total }}$ & $t_{\text {epoch }}$ & |\#Epoch & $t_{\text {total }}$ & $t_{\text {epoch }}$ \\
\hline ULMFiT & 11 & $11 \mathrm{~min}$ & $1 \mathrm{~min}$ & 11 & $99 \mathrm{~min}$ & $9 \mathrm{~min}$ \\
\hline biLM(ELMo) & 5 & $25 \min$ & $5 \min$ & 2 & $64 \mathrm{~min}$ & $32 \mathrm{~min}$ \\
\hline GPT $s e q_{\max }=256$ & 3 & $57 \min$ & $19 \min$ & 3 & $90 \mathrm{~min}$ & $30 \mathrm{~min}$ \\
\hline BERT $s e q_{\max }=256$ & 3 & $36 \mathrm{~min}$ & $12 \mathrm{~min}$ & 2 & $38 \mathrm{~min}$ & $19 \min$ \\
\hline
\end{tabular}

\subsection{Classifier Fine-tuning}

The results of the downstream classification tasks are shown in Table 7 BERT with our pretraining data outperforms all existing models on the private set of Wongnai and Wisesight and obtains $0.9 \%$ and $3.2 \%$ respective absolute accuracy improvement over the state-of-the-art. Absolute accuracy improvements on all models and tasks are obtained when pretrained with our Thai Social Media data instead of the Thai Wiki Dump.

\subsection{Limited Pretraining Corpus}

We also investigated the performance of the models in the scenario where the pretraining corpus is limited. This result reflects the learning ability of the models in a language where training data is scarce. We randomly sampled a total of $40 \mathrm{M}$ tokens (equivalent to around $234 \mathrm{~K}$ threads) from the dataset used in our previous experiments. ULMFiT, ELMo, and GPT are trained for 3 epochs while BERT is trained for 30k steps (equivalent to approximately 6.5 epochs on this data). Table 8 shows that ULMFiT and GPT perform considerably well. On the other hand, adding ELMo to LSTM input shows little improvement. This means that ELMo requires a larger corpus to be effective. Although BERT performs well on the Wisesight dataset, it has a drop in performance on Wongnai dataset.

\section{Conclusion}

Our work shows that by using our unique data preprocessing methods and our pretraining social media data, we can improve the performance of the LMs in the downstream tasks. The improvement of all models from pretraining data 
Table 7. Classifier Fine-tuning Results. Our models are compared to other models: the baseline that predicts the most frequent label, the latest Kaggle competition winner, and public github repositories. The public leaderboard and private leaderboard are calculated with approximately $30 \%$ and $70 \%$ of the test data respectively.

\begin{tabular}{l|cc|cc}
\hline Model & \multicolumn{3}{|c}{ Wisesight (Acc.) } & \multicolumn{2}{c}{ Wongnai (Acc.) } \\
& Private & Public & Private & Public \\
\hline Baseline & 0.5809 & 0.6044 & 0.4785 & 0.4785 \\
Kaggle Best & 0.7597 & 0.7532 & 0.5914 & 0.5814 \\
fastText [3] & 0.6131 & 0.6314 & 0.5145 & 0.5109 \\
LinearSVC [3] & - & - & 0.5022 & 0.4976 \\
Logistic Regression [3] & 0.7499 & 0.7278 & - & - \\
\hline Thai Wiki Dump Pretraining & & & \\
ULMFiT [3] & 0.7419 & 0.7126 & 0.5931 & 0.6032 \\
ULMFiT Semi-supervised [3] & 0.7597 & 0.7337 & - & - \\
BERT seqmax $=128$ [1] & - & - & 0.5661 & 0.5706 \\
\hline Ours (Thai Social Media & Pretraining) & & \\
ULMFiT & 0.7586 & 0.7346 & 0.6203 & $\mathbf{0 . 6 4 0 9}$ \\
biLSTM & 0.6366 & 0.6213 & 0.4773 & 0.4946 \\
ELMo+biLSTM & 0.6866 & 0.6450 & 0.5310 & 0.5226 \\
GPT seqmax $=256$ & 0.7669 & $\mathbf{0 . 7 5 4 0}$ & 0.6088 & 0.6145 \\
BERT seqmax $=256$ & $\mathbf{0 . 7 6 9 1}$ & 0.7439 & $\mathbf{0 . 6 2 5 1}$ & 0.6231 \\
\hline
\end{tabular}

Table 8. Limited Pretraining Corpus Results. The public and private scores are calculated with approximately $30 \%$ and $70 \%$ of the test data respectively.

\begin{tabular}{|c|c|c|c|c|}
\hline \multirow[t]{2}{*}{ Model } & \multicolumn{2}{|c|}{ Wisesight (Acc.) } & \multicolumn{2}{|c|}{ Wongnai (Acc.) } \\
\hline & Private & Public| & Private & Public \\
\hline ULMFiT & 0.7358 & 0.7143 & 0.5984 & 0.6290 \\
\hline biLSTM & 0.6366 & 0.6213 & 0.4773 & 0.4946 \\
\hline biLSTM + ELMo & 0.6489 & 0.6095 & 0.4879 & 0.4753 \\
\hline GPT $s e q_{\max }=256$ & 0.6931 & 0.7075 & 0.6111 & 0.6102 \\
\hline BERT $s e q_{\max }=256$ & 0.7467 & 0.7244 & 0.5650 & 0.5516 \\
\hline
\end{tabular}

of the same domain suggests that pretraining data has a significant impact on LM performance. Moreover, the possibility for LM pretraining on a noisy corpus shows the ability of the models to learn in spite of the quality of the data.

Results-wise, BERT is the best performing model with respect to classification accuracy. It can achieve state-of-the-art results on both of the benchmarking downstream tasks. However, it has unstable performance on downstream tasks when pretrained on a small corpus and uses a lot of pretraining time. If speed and ease of training are the main considerations, we recommend using AWD-LSTM with ULMFiT due to its speed of pretraining and fine-tuning, while the results are still on par with transformer-based models. Although OpenAI GPT shows promising results with acceptable pretraining speed, it is overshadowed by other models in both aspects. Finally, although ELMo shows significant improvements 
when compared with the baseline biLSTM, it places a dependency on designing a powerful task-specific model to achieve good performance.

\section{Acknowledgements}

In the making of the paper, the authors would like to acknowledge Mr. Can Udomcharoenchaikit for his continuous and insightful research suggestions until the completion of this paper.

\section{References}

1. Bert-th. https://github.com/ThAIKeras/bert (2019)

2. scrapy. https://github.com/scrapy/scrapy (2019)

3. thai2fit. https://github.com/cstorm125/thai2fit (2019)

4. Aroonmanakun, W.: Thoughts on word and sentence segmentation in thai (2007)

5. Bahdanau, D., Cho, K., Bengio, Y.: Neural machine translation by jointly learning to align and translate. arXiv preprint arXiv:1409.0473 (2014)

6. Cho, K., van Merrienboer, B., Gulcehre, C., Bahdanau, D., Bougares, F., Schwenk, H., Bengio, Y.: Learning Phrase Representations using RNN Encoder-Decoder for Statistical Machine Translation. arXiv e-prints arXiv:1406.1078 (Jun 2014)

7. Dai, A.M., Le, Q.V.: Semi-supervised Sequence Learning. arXiv e-prints arXiv:1511.01432 (Nov 2015)

8. Devlin, J., Chang, M.W., Lee, K., Toutanova, K.: BERT: Pre-training of Deep Bidirectional Transformers for Language Understanding. arXiv e-prints arXiv:1810.04805 (Oct 2018)

9. Howard, J., Ruder, S.: Universal Language Model Fine-tuning for Text Classification. arXiv e-prints arXiv:1801.06146 (Jan 2018)

10. Lertpiya, A., Chaiwachirasak, T., Maharattanamalai, N., Lapjaturapit, T., Chalothorn, T., Tirasaroj, N., Chuangsuwanich, E.: A preliminary study on fundamental thai nlp tasks for user-generated web content. In: 2018 International Joint Symposium on Artificial Intelligence and Natural Language Processing (iSAINLP). pp. 1-8 (Nov 2018). https://doi.org/10.1109/iSAI-NLP.2018.8692946

11. Merity, S., Shirish Keskar, N., Socher, R.: Regularizing and Optimizing LSTM Language Models. arXiv e-prints arXiv:1708.02182 (Aug 2017)

12. Mikolov, T., Chen, K., Corrado, G., Dean, J.: Efficient Estimation of Word Representations in Vector Space. arXiv e-prints arXiv:1301.3781 (Jan 2013)

13. Pennington, J., Socher, R., Manning, C.D.: Glove: Global vectors for word representation. In: Empirical Methods in Natural Language Processing (EMNLP). p. 1532-1543 (2014)

14. Peters, M.E., Neumann, M., Iyyer, M., Gardner, M., Clark, C., Lee, K., Zettlemoyer, L.: Deep contextualized word representations. arXiv e-prints arXiv:1802.05365 (Feb 2018)

15. Pythainlp 2.0. https://github.com/PyThaiNLP/pythainlp (2019)

16. Radford, A., Narasimhan, K., Salimans, T., Sutskever, I.: Improving language understanding by generative pre-training (2018)

17. Sutskever, I., Vinyals, O., Le, Q.V.: Sequence to Sequence Learning with Neural Networks. arXiv e-prints arXiv:1409.3215 (Sep 2014)

18. Vaswani, A., Shazeer, N., Parmar, N., Uszkoreit, J., Jones, L., Gomez, A.N., Kaiser, L., Polosukhin, I.: Attention Is All You Need. arXiv e-prints arXiv:1706.03762 (Jun 2017) 